\title{
INVERSE PROBLEMS OF THE CALCULUS OF VARIATIONS FOR MULTIPLE INTEGRALS ${ }^{1}$
}

\author{
WILLIAM A. PATTERSON
}

1. Introduction. The simplest case of the inverse problem of Darboux is that in which an ordinary differential equation in the normal form $y^{\prime \prime}=\phi\left(x, y, y^{\prime}\right)$ is assigned with the requirement that we ascertain, first, under what conditions $\phi\left(x, y, y^{\prime}\right)$ is the solution for $y^{\prime \prime}$ of the Euler equation of a variation problem of the form $\int_{x_{1}}^{x_{2}} f\left(x, y, y^{\prime}\right) d x$ $=\min$ and then that we determine the most general integrand function $f$ corresponding to an admissible function ${ }^{2} \phi\left(x, y, y^{\prime}\right)$.

For partial differential equations the simplest analogous problem is that of finding the most general first order multiple integral variation problem associated with an assigned partial differential equation, that is, the most general integrand function $f$ of a variation problem of the form

$$
\int_{(n)} f\left(x_{1}, \cdots, x_{n}, z, p_{1}, \cdots, p_{n}\right) d x_{1} \cdots d x_{n}=\min , p_{i}=\partial z / \partial x_{i},
$$

of which the extremal hypersurfaces are the integral hypersurfaces $z=z\left(x_{1}, \cdots, x_{n}\right)$ of a prescribed partial differential equation.

A systematic study of such inverse problems of Darboux type for certain important classes of partial differential equations is made in this paper.

2. A uniqueness theorem. Consider a partial differential equation of the form

$$
\begin{aligned}
F \equiv & A_{\alpha \beta}\left(x_{1}, \cdots, x_{n}, z, p_{1}, \cdots, p_{n}\right) p_{\alpha \beta} \\
& +B\left(x_{1}, \cdots, x_{n}, z, p_{1}, \cdots, p_{n}\right)=0
\end{aligned}
$$

where $p_{i j}=\partial^{2} z / \partial x_{i} \partial x_{j}$, and $A_{i j}=A_{j i}(i, j=1, \cdots, n)$ and $B$ are arbitrary analytic functions of $x_{1}, \cdots, x_{n}, z, p_{1}, \cdots, p_{n}$. In (2.1) as elsewhere in this paper, a repeated Greek letter is an umbral index indicating a summation with range 1 to $n$, unless otherwise indicated.

Equation (2.1), as it stands, may have an equation of variation which is self-adjoint on every hypersurface $z=z\left(x_{1}, \cdots, x_{n}\right)$. If so there is always a multiple integral of the form (I) having $F=0$ as its

\footnotetext{
1 Presented to the Society, December 29, 1939.

${ }^{2}$ Cf. G. Darboux, Theorie des Surfaces, vol. 3, 1887, p. 53. For the case of $n \geqq 2$ dependent variables $y_{1}, \cdots, y_{n}$ see L. LaPaz, Proceedings of the National Academy of Sciences, vol. 17 (1931), pp. 459-463.
} 
Euler-Lagrange equation. The most general such integral is known to have an integrand function of the form ${ }^{3}$

$$
f=G+C+D_{\alpha} p_{\alpha}+\partial \omega_{\alpha} / \partial x_{\alpha},
$$

where $G\left(x_{1}, \cdots, x_{n}, z, p_{1}, \cdots, p_{n}\right)$ is a particular solution of

$$
g_{p_{i} p_{j}}=A_{i j}, \quad i, j=1, \cdots, n,
$$

where the set $\left\{C\left(x_{1}, \cdots, x_{n}, z\right), D_{i}\left(x_{1}, \cdots, x_{n}, z\right)\right\}$ is a particular solution of

$$
C_{z}-D_{\alpha x_{\alpha}}=G_{p_{\alpha} x_{\alpha}}+G_{p_{\alpha} z} p_{\alpha}-G_{z}-B,
$$

and where the $\omega_{i}$ occurring in the partial derivatives with respect to $x_{i}$ are arbitrary functions of $x_{1}, \cdots, x_{n}, z$ alone. Whether (2.1), as it stands, has a self-adjoint equation of variation or not, there may exist equivalent equations which do have self-adjoint equations of variation. We proceed to the question of the existence of such equivalent equations.

For a problem of minimizing the multiple integral (I), the Lagrange partial differential equation must necessarily be a second order partial differential equation ${ }^{4}$ linear in the $p_{i j}$ and with a self-adjoint equation of variation. Thus, if the integral hypersurfaces $z=z\left(x_{1}, \cdots, x_{n}\right)$ of $F=0$ are to be the extremal hypersurfaces of such a problem, the partial differential equation $F=0$ must be equivalent to a partial differential equation of the second order with the properties of linearity and self-adjointness of the equation of variation just described. Since the analytic partial differential equation $F=0$ is itself linear in the $p_{i j}$, it follows that the most general linear equation

$a_{\mu \nu}\left(x_{1}, \cdots, x_{n}, z, p_{1}, \cdots, p_{n}\right) p_{\mu \nu}+b\left(x_{1}, \cdots, x_{n}, z, p_{1}, \cdots, p_{n}\right)=0$

equivalent to $F=0$ is of the form $M \cdot F=0$, where $M \neq 0$ is a function of $x_{1}, \cdots, x_{n}, z, p_{1}, \cdots, p_{n}$ alone. A function $M\left(x_{1}, \cdots, x_{n}, z, p_{1}, \cdots, p_{n}\right)$ $\neq 0$ and such that the equation $M \cdot F=0$ has a self-adjoint equation of variation will be called a multiplier of the partial differential equation $F=0$. Since multipliers which differ only by a nonzero constant factor are not regarded as distinct, it is permissible to restrict attention to multipliers $M>0$.

If the equation $M \cdot F=0$ is to have a self-adjoint equation of variation, then the multiplier $M$ must satisfy the following relations ${ }^{5}$ identically in the variables $x_{1}, \cdots, x_{n}, z, p_{1}, \cdots, p_{n}$ :

${ }^{3}$ Cf. L. LaPaz, Transactions of this Society, vol. 32 (1930), p. 513.

${ }^{4}$ Integrand functions linear in the $p_{i}$ are excluded from consideration.

${ }^{5}$ L. LaPaz, loc. cit., p. 512. 


$$
\begin{array}{rr}
M \cdot A_{i j}=M \cdot A_{j i}, & i, j=1,2, \cdots, n \\
\partial\left(M \cdot A_{i j}\right) / \partial p_{k}=\partial\left(M \cdot A_{k j}\right) / \partial p_{i}, & i, j, k=1,2, \cdots, n ; k \neq i, \\
\partial\left(M \cdot A_{i \alpha}\right) / \partial x_{\alpha}+p_{\alpha} \partial\left(M \cdot A_{i \alpha}\right) / \partial z-\partial(M \cdot B) / \partial p_{i} & =0, \\
i & =1,2, \cdots, n .
\end{array}
$$

It is evident that the conditions (2.5) are automatically satisfied. Hence if a multiplier $M\left(x_{1}, \cdots, x_{n}, z, p_{1}, \cdots, p_{n}\right)$ exists, it must satisfy the relations (2.6) which may be written in the form

$$
\begin{aligned}
A_{i j} \partial M / \partial p_{k}-A_{k j} \partial M / \partial p_{i}+\left(\partial A_{i j} / \partial p_{k}-\partial A_{k j} / \partial p_{i}\right) \cdot M & =0 \\
A_{i \alpha} \partial M / \partial x_{\alpha}+ & p_{\alpha} \cdot A_{i \alpha} \partial M / \partial z-B \cdot \partial M / \partial p_{i} \\
& \quad+\left(\partial A_{i \alpha} / \partial x_{\alpha}+p_{\alpha} \partial A_{i \alpha} / \partial z-\partial B / \partial p_{i}\right) \cdot M=0 .
\end{aligned}
$$

If $F=0$, as it stands, has a self-adjoint equation of variation, then (2.7) reduces to

$$
\begin{aligned}
A_{i j} \partial M / \partial p_{k}-A_{k j} \partial M / \partial p_{i} & =0, \\
A_{i \alpha} \partial M / \partial x_{\alpha}+p_{\alpha} \cdot A_{i \alpha} \partial M / \partial z-B \partial M / \partial p_{i} & =0 .
\end{aligned}
$$

If $a$, the determinant of the matrix $\left(A_{i j}\right)$, is different from zero, it follows from $\left(2.8_{1}\right)$ that $\partial M / \partial p_{i}=0(i=1,2, \cdots, n)$. But then $\left(2.8_{2}\right)$ is seen to imply that $\partial M / \partial x_{i}=\partial M / \partial z=0(i=1,2, \cdots, n)$; that is to say, $M$ is a constant. This completes the proof of the following result.

THEOREM 2.1. If $F=0$, as it stands, has a self-adjoint equation of variation in a neighborhood in which $\mathfrak{a} \neq 0$, then $F=0$ admits a constant as its most general multiplier in this neighborhood.

As an immediate corollary to Theorem 2.1 we have the following important uniqueness theorem.

TheOREM 2.2. In a neighborhood in which $a \neq 0$, the equation $F=0$ admits at most one multiplier $M$.

3. Variation problems in $(n+1)$-space of which the extremals are minimal hypersurfaces. As an interesting application of Theorem 2.2, let us consider the inverse problem of Darboux for minimal hypersurfaces ${ }^{6}$ in $(n+1)$-space $(n>2)$. The corresponding inverse problem for straight lines in $(n+1)$-space $(n>2)$, that is, for solution curves of the system of differential equations $y_{i}{ }^{\prime \prime}=0(i=1,2, \cdots, n)$ has recently been treated by $D$. R. Davis ${ }^{7}$ who has found that the most general integrand function $f\left(x, y_{1}, \cdots, y_{n}, y_{1}{ }^{\prime}, \cdots, y_{n}{ }^{\prime}\right)$ of a varia-

${ }^{6}$ For the case $n=2$ see L. LaPaz, Acta Szeged, vol. 5 (1932), pp. 199-207.

7 D. R. Davis, Transactions of this Society, vol. 33 (1931), pp. 244-251. 
tion problem of the form $\int_{x_{1}}^{x_{2}} f d x=$ min for which the extremals are the solution curves of the system of differential equations $y_{i}^{\prime \prime}=0$ $(i=1,2, \cdots, n)$ is of the form $f=g\left(x, y_{1}, \cdots, y_{n}, y_{1}{ }^{\prime}, \cdots, y_{n}{ }^{\prime}\right)$ $+d t\left(x, y_{1}, \cdots, y_{n}\right) / d x$, where $g$ is a suitably chosen particular solution of a (compatible) system of second order partial differential equations, with $i<j=1,2, \cdots, n$, of the form

$$
\partial^{2} g / \partial y_{i}^{\prime} \partial y_{j}^{\prime}=P_{i j}\left(y_{1}^{\prime}, \cdots, y_{n}^{\prime}, y_{1}-y_{1}^{\prime} x, \cdots, y_{n}-y_{n}^{\prime} x\right),
$$

and where $t$ is an arbitrary function of its arguments. The functions $P_{i j}$ are arbitrary solutions of a certain system of partial differential equations considered by Davis. In sharp contrast to this result we shall show that the most general variation problem (I) for which the extremals are minimal hypersurfaces in the sense defined below is uniquely determined up to an additive function of the form $\partial \omega_{\alpha} / \partial x_{\alpha}$ where the $\omega_{i}(i=1,2, \cdots, n)$ are arbitrary functions of $x_{1}, \cdots, x_{n}, z$.

A surface $z=z\left(x_{1}, \cdots, x_{n}\right)$ defined and of class $C^{\prime \prime}$ in a region $R$ of $\left(x_{1}, \cdots, x_{n}\right)$-space will be called a minimal hypersurface if $z$ and its partial derivatives of the first and second orders satisfy in $R$ the partial differential equation

$$
F_{1} \equiv\left[\left(1+p_{\gamma} p_{\gamma}\right) \delta_{\alpha}^{\beta}-p_{\alpha} p_{\beta}\right] \cdot p_{\alpha \beta}=0,
$$

where $\delta_{j}^{i}$ is Kronècker's delta. $F_{1}=0$ is of the form (2.1) with $a \equiv\left(1+p_{\gamma} p_{\gamma}\right)^{n-1} \neq 0$ and therefore by Theorem 2.2 there exists at most one multiplier $M$ for $F_{1}=0$. But the Lagrange partial differential equation for the problem $\int_{(n)}\left(1+p_{\gamma} p_{\gamma}\right)^{1 / 2} d x_{1} \cdots d x_{n}=\min$ is of the form $M \cdot F_{1}=0$ where $M=\left(1+p_{\gamma} p_{\gamma}\right)^{-3 / 2}$. Hence, calculating the integrand function $f$ of (2.2), we reach the following conclusion.

THEOREM 3.1. The partial differential equation $F_{1}=0$ of minimal hypersurfaces admits the unique multiplier $M=\left(1+p_{\gamma} p_{\gamma}\right)^{-3 / 2}$. The most general variation problem (I) for which the extremal hypersurfaces are minimal hypersurfaces has an integrand function $f$ of the form $f=\left(1+p_{\gamma} p_{\gamma}\right)^{1 / 2}+\partial \omega_{\alpha} / \partial x_{\alpha}$, where the $\omega_{i}$ are arbitrary functions of $x_{1}, \cdots, x_{n}, z$ alone.

4. Variation problems associated with the equation $A_{\alpha \beta} p_{\alpha \beta}$ $+B\left(x_{1}, \cdots, x_{n}, z\right)=0$. In this section we shall consider the problem of finding the most general variation problem (I) associated with the partial differential equation

$$
F_{2} \equiv A_{\alpha \beta} p_{\alpha \beta}+B\left(x_{1}, \cdots, x_{n}, z\right)=0,
$$

where $A_{i j}=A_{j i}(i, j=1,2, \cdots, n)$ are real constants not all zero and $B$ is an arbitrary analytic function of $x_{1}, \cdots, x_{n}, z$. 
Partial differential equations of the form (4.1) are of much importance in pure and applied mathematics. For example if we choose $n=2, A_{i j}=\delta_{j}^{i}$ and $B=8 z /\left(1+x_{1}^{2}+x_{2}^{2}\right)^{2}$, (4.1) reduces to the partial differential equation studied by Schwarz in his researches on minimal surfaces. For the same value of $n$ and suitable choice of $A_{i j}$ and $B$, the equation (4.1), with appropriate interpretation of the variables $x_{1}, x_{2}$ and $z$, includes many of the most important partial differential equations of mathematical physics.

It is easy to verify that all equations (4.1) have self-adjoint equations of variation and hence, as they stand, are Euler-Lagrange equations. We therefore proceed immediately to the problem of determining the most general integrand function $f$ of the problem (I) known to be associated with an equation of the form $F_{2}=0$. Application of Theorem 2.1 and calculation of the function $f$ of (2.2) leads to the following theorem.

THEOREM 4.1. If $\mathfrak{a} \neq 0$, then the partial differential equation $F_{2}=0$ admits a constant as its most general multiplier. The most general variation problem (I) associated with $F_{2}=0$ has an integrand function $f$ of the form $f=\frac{1}{2} A_{\alpha \beta} p_{\alpha} p_{\beta}-\int_{z_{0}}^{z} B d z+\partial \omega_{\alpha} / \partial x_{\alpha}$, where the $\omega_{i}$ are arbitrary functions of $x_{1}, \cdots, x_{n}, z$.

As an application of this case we find that the most general variation problem (I) associated with $s=\partial^{2} z / \partial x_{1} \partial x_{2}=0$, which is of the type $F_{2}=0$ with $n=2$ and $B=0$, has an integrand function of the form $f=p_{1} p_{2} / 2+\partial \omega_{1} / \partial x_{1}+\partial \omega_{2} / \partial x_{2}$ where $\omega_{1}, \omega_{2}$ are arbitrary functions of $x_{1}, x_{2}, z$.

If $\mathfrak{a}=0$ our discussion proceeds on the basis of the system (2.8) written for the equation $F_{2}=0$. It is evident that in every case the resulting system of equations $S$ can be replaced by an equivalent system $S^{*}$ of $R \leqq 2 n+1$ independent partial differential equations. ${ }^{8}$ The number $R$ of equations in $S^{*}$ is equal to the rank of $(A)$, the matrix of coefficients of the system $S$. Inspection of $(A)$ discloses that its last column is a linear combination of the $n$ preceding columns. Thus $R$ is always less than $2 n+1$. Actually, when $\mathfrak{a}=0, R$ is at most $2 n-1$ and its precise value is given by the following lemmas which we state without proof. ${ }^{9}$

${ }^{8}$ Cf. E. Goursat, Léçons sur l'Intégration des Équations aux Dérivées Partielles du Premier Ordre, 1921, p. 66.

9 These proofs and other details omitted in this paper are to be found in the writer's doctoral dissertation, Inverse Problems of the Calculus of Variations for Multiple Integrals, The Ohio State University, 1936. For assistance in the preparation of this dissertation and the present paper, the writer wishes to express his indebtedness to Professor Lincoln LaPaz of The Ohio State University. 
LEMma 1. If $\left(A_{i j}\right)$ is of rank $r(r=2,3, \cdots, n-1)$, the matrix $(A)$ is of rank $n+r$.

LEMma 2. If $\left(A_{i j}\right)$ is of rank 1 , the matrix $(A)$ is of rank $n$.

Where it is desirable to retain the original variables $x_{i}$, a straightforward but somewhat involved discussion based on Lemmas 1 and 2 enables us to treat ${ }^{10}$ both the case $1<r<n$ and the case $r=1$. We give a simpler treatment of the cases $1 \leqq r<n$ using another lemma.

LEMMA 3. If $\left(A_{i j}\right)$ is of rank $r$, then by means of a nonsingular linear transformation $X_{i}=c_{i \alpha} x_{\alpha}(i=1,2, \cdots, n)$, where the $c_{i j}$ are constants, the partial differential equation $F_{2}=0$ can be transformed into one such that $^{11} A_{i j}=\delta_{j}^{i}, A_{i i}= \pm 1(i \leqq r), A_{i i}=0(i>r)$.

By use of Lemma 3, (2.8) can be replaced by the equivalent syste $m$ (4.2) $A_{i i} \partial M / \partial p_{k}-A_{k i} \partial M / \partial p_{i}=0, i=1,2, \cdots, r ; k=1,2, \cdots, n$, (4.3) $A_{i i}\left(\partial M / \partial x_{i}+p_{i} \partial M / \partial z\right)-B \partial M / \partial p_{i}=0, \quad i=1,2, \cdots, n$. From (4.2) with $i=1$ and $k>1$ we infer $\partial M / \partial p_{k}=0(k>1)$. If now $1<r<n$, the choice $i=2, k=1$ shows $\partial M / \partial p_{1}=0$. Hence for $1<r<n$ it follows from $\partial M / \partial p_{i}=0 \quad(i=1,2, \cdots, n)$ and $(4.3)$ that $\partial M / \partial x_{i}+p_{i} \partial M / \partial z=0(i \leqq r)$ and therefore that $\partial M / \partial x_{i}=\partial M / \partial z=0$ $(i \leqq r)$. This completes the proof of the following theorem.

TheOREM 4.2. If the rank of $\left(A_{i j}\right)$ is $r(1<r<n)$, then the partial differential equation $F_{2}=0$, when reduced to the normal form of Lemma 3, admits as its most general multiplier the function $A\left(x_{r+1}, x_{r+2}, \cdots, x_{n}\right)$ where $A$ is different from zero but is otherwise an arbitrary function of its arguments.

We now proceed to calculate the function $f$ of (2.2). A particular solution of (2.3) formed for the equation $A\left(x_{r+1}, x_{r+2}, \cdots, x_{n}\right) \cdot F_{2}=0$ is $G=\frac{1}{2} A\left(x_{r+1}, x_{r+2}, \cdots, x_{n}\right) A_{\mu \mu} p_{\mu} p_{\mu}$. Substituting this value of $G$ in (2.4), we have

$$
C_{z}-D_{\alpha x_{\alpha}}=-B \cdot A\left(x_{r+1}, x_{r+2}, \cdots, x_{n}\right) .
$$

A particular solution of this equation is $D_{i}=0(i=1,2, \cdots, n)$, $C=-A\left(x_{r+1}, x_{r+2}, \cdots, x_{n}\right) \cdot \int_{z_{0}}^{z} B d z$. We thus get the following result.

TheOREM 4.3. If the rank of $A_{i j}$ is $r(1<r<n)$, then the most general variation problem (I) associated with the equation $F_{2}=0$, when reduced to the normal form of Lemma 3, has an integrand function $f$ of the form

10 See the dissertation cited in footnote 9.

${ }^{11}$ Cf. L. E. Dickson, Modern Algebraic Theories, 1926, pp. 71-72. 


$$
f=A\left(x_{r+1}, x_{r+2}, \cdots, x_{n}\right) \cdot\left[\frac{A_{\mu \mu} p_{\mu} p_{\mu}}{2}-\int_{z_{0}}^{z} B d z\right]+\partial \omega_{\alpha} / \partial x_{\alpha}
$$

where the $\omega_{i}$ are arbitrary functions of $x_{1}, x_{2}, \cdots, x_{n}, z$.

Finally if the rank of $\left(A_{i j}\right)$ is $r=1$, then $\partial M / \partial p_{k}=0$ for all $k>1$ and (4.3) reduces to the single equation $A_{11}\left(\partial M / \partial x_{1}+p_{1} \partial M / \partial z\right)$ $=B \cdot \partial M / \partial p_{1}$, which obviously has two independent solutions $M_{1}\left(x_{1}, z, p_{1}\right)$ and $M_{2}\left(x_{1}, z, p_{1}\right)$ for $M$. In this case we are thus led to a somewhat different result.

TheOREm 4.4. If the rank of $\left(A_{i j}\right)$ is 1 , then the partial differential equation $F_{2}=0$, when reduced to the normal form of Lemma 3, admits as its most general multiplier the functions $A\left(M_{1}, M_{2}, x_{2}, x_{3}, \cdots, x_{n}\right)$ where $A$ is different from zero but is otherwise an arbitrary function.

The determination of the most general integrand function $f$ when $r=1$ may be left to the reader.

As an application of this case we find that the most general multiplier for $r=\partial^{2} z / \partial x_{1}^{2}=0$, which is of type $F_{2}=0$ with $n=2$ and $B=0$, is $A\left(p_{1}, z-p_{1} x_{1}, x_{2}\right)$. The most general variation problem (I) associated with $r=0$ has an integrand function $f$ of the form

$$
f=\int_{p_{1_{0}}}^{p_{1}} \int_{p_{1_{0}}}^{p_{1}} A\left(p_{1}, z-p_{1} x_{1}, x_{2}\right) d p_{1} d p_{1}+\partial \omega_{1} / \partial x_{1}+\partial \omega_{2} / \partial x_{2}
$$

where $\omega_{1}, \omega_{2}$ are arbitrary functions of $x_{1}, x_{2}, z$.

5. Variation problems associated with the equation $R(p, q) r$ $+2 S(p, q) s+T(p, q) t=0$. Consider the partial differential equation

$$
F_{3} \equiv R(p, q) r+2 S(p, q) s+T(p, q) t=0,
$$

where $z=z(x, y), \quad p=\partial z / \partial x, q=\partial z / \partial y, \quad r=\partial^{2} z / \partial x^{2}, \quad s=\partial^{2} z / \partial x \partial y$, $t=\partial^{2} z / \partial y^{2}$, and the coefficients $R, S, T$ are analytic functions of $p$ and $q$. We shall exclude equations for which two of the coefficients $R, S, T$ vanish identically, since such vanishing would reduce the equation $F_{3}=0$ to one of the special equations $r=0, s=0$ treated in the previous section. The system (2.7) with $n=2$ and suitable identification of the variables now has the form

$$
\begin{aligned}
S \cdot \partial M / \partial p-R \cdot \partial M / \partial q+M \cdot[\partial S / \partial p-\partial R / \partial q] & =0, \\
T \cdot \partial M / \partial p-S \cdot \partial M / \partial q+M \cdot[\partial T / \partial p-\partial S / \partial q] & =0, \\
R \cdot[\partial M / \partial x+p \partial M / \partial z]+S \cdot[\partial M / \partial y+q \partial M / \partial z] & =0, \\
S \cdot[\partial M / \partial x+p \partial M / \partial z]+T \cdot[\partial M / \partial y+q \partial M / \partial z] & =0 .
\end{aligned}
$$


In contradistinction to (4.1), (5.1) may (Case I) or may not (Case II) have a self-adjoint equation of variation. In Case I, since $\partial S / \partial p$ $-\partial R / \partial q=\partial T / \partial p-\partial S / \partial q=0,(5.2)$ becomes the homogeneous system

$$
\begin{aligned}
& U_{1} M \equiv S \cdot \partial M / \partial p-R \cdot \partial M / \partial q=0 \\
& U_{2} M \equiv T \cdot \partial M / \partial p-S \cdot \partial M / \partial q=0, \\
& U_{3} M \equiv R \cdot[\partial M / \partial x+p \cdot \partial M / \partial z]+S \cdot[\partial M / \partial y+q \cdot \partial M / \partial z]=0 \\
& U_{4} M \equiv S \cdot[\partial M / \partial x+p \cdot \partial M / \partial z]+T \cdot[\partial M / \partial y+q \cdot \partial M / \partial z]=0 .
\end{aligned}
$$

In either case the equations of (5.2) and (5.3) are found to be independent if and only if $\delta \equiv R T-S^{2} \neq 0$. Each of the Cases I, II may therefore be further subdivided according as (1) $\delta \neq 0$, (2) $\delta \equiv 0$. We proceed to a discussion of these cases.

Case $\mathrm{I}_{1}$. When (5.1) has a self-adjoint equation of variation and $\delta \neq 0$, it follows from Theorem 2.1 that $F_{3}=0$ has a constant as its most general multiplier. Hence we have proved the following.

THEOREM 5.1. If the equation $F_{3}=0$ has a self-adjoint equation of variation in a neighborhood in which $\delta \neq 0$, then the most general variation problem (I) associated with $F_{3}=0$ in this neighborhood has an integrand function $f$ of the form $f=G+\partial \omega_{1} / \partial x+\partial \omega_{2} / \partial y$, where $G(x, y, z, p, q)$ is a particular solution of $g_{p p}=R, g_{p q}=S, g_{q q}=T$, satisfying $G_{p x}+G_{q y}$ $+p G_{p z}+q G_{q z}-G_{z}=0$ and the $\omega_{i}$ are arbitrary functions of $x, y, z$.

As an illustration we note that the most general variation problem (I) associated with the equation $q^{2} r+4 p q s+p^{2} t=0$ has an integrand function of the form $f=p^{2} q^{2} / 2+\partial \omega_{1} / \partial x+\partial \omega_{2} / \partial y$, where the $\omega_{i}$ are arbitrary functions of $x, y, z$.

Case $\mathrm{I}_{2}$. When $\delta \equiv 0$ it follows that $R \cdot S \cdot T \not \equiv 0$ and hence, in any neighborhood in which $R \cdot S \cdot T \neq 0, U_{k+1} M \equiv(T / S) \cdot U_{k} M(k=1,3)$. The system $U_{i} M=0(i=1,3)$ is therefore equivalent to (5.3) and moreover is found to be complete. Hence we have the following result.

THEOREM 5.2. If $F_{3}=0$ has a self-adjoint equation of variation in a neighborhood in which $R \cdot S \cdot T \neq 0$ and $\delta \equiv 0$, then it admits as its most general multiplier in this neighborhood the function $M(x, y, z, p, q)$ $=A\left(M_{1}, M_{2}, M_{3}\right)$ where $M_{i}(i=1,2,3)$ are any three independent integrals of $U_{i} M=0(i=1,3)$ and $A \neq 0$ is an arbitrary function.

The most general variation problem (I) can now be obtained in the usual manner. The equation $\phi(p+q) \cdot[r+2 s+t]=0$, where $\phi$ is an arbitrary analytic function of its argument, illustrates this case.

We now take up Case II in which $F_{3}=0$, as it stands, does not have a self-adjoint equation of variation. If in addition $\delta \neq 0$, then we can 
infer from Theorem 2.2 that at most one multiplier $M(x, y, z, p, q)$ exists. To secure more precise information in this case we must discuss the nonhomogeneous system (5.2). To integrate (5.2) we transform it into a system linear and homogeneous in the first partial derivatives of a function $m(x, y, z, p, q, M)$ with $\partial m / \partial M \neq 0$, which defines $M$ by means of the relation $m=$ const. The resulting system

$$
\begin{aligned}
& U_{1} m \equiv S \partial m / \partial p-R \partial m / \partial q-M \cdot[\partial S / \partial p-\partial R / \partial q] \cdot \partial m / \partial M=0, \\
& U_{2} m \equiv T \partial m / \partial p-S \partial m / \partial q-M \cdot[\partial T / \partial p-\partial S / \partial q] \cdot \partial m / \partial M=0, \\
& U_{3} m \equiv R(\partial m / \partial x+p \partial m / \partial z)+S(\partial m / \partial y+q \partial m / \partial z)=0, \\
& U_{4} m \equiv S(\partial m / \partial x+p \partial m / \partial z)+T(\partial m / \partial y+q \partial m / \partial z)=0
\end{aligned}
$$

is independent since $\delta \neq 0$. It is easy to verify that the commutator $\left(U_{1} U_{4}\right) m \equiv U_{5} m$ is not a linear combination of $U_{i} m(i=1,2,3,4)$ and we accordingly adjoin $U_{5} m=0$ to the system (5.4). It is next found that the commutator $\left(U_{1} U_{2}\right) m$ is a linear combination of $U_{i} m$ $(i=1,2,3,4,5)$ if and only if the following determinant $D$ vanishes:

$\left|\begin{array}{ccc}S & T & S \cdot[\partial T / \partial p+\partial S / \partial q]-R \cdot \partial T / \partial q-T \cdot \partial S / \partial p \\ R & S & S \cdot[\partial S / \partial p+\partial R / \partial q]-R \cdot \partial S / \partial q-T \cdot \partial R / \partial p \\ \partial S / \partial p-\partial R / \partial q & \partial T / \partial p-\partial S / \partial q & V\end{array}\right|$

where

$$
\begin{aligned}
V= & S \cdot\left[\partial^{2} T / \partial p^{2}-\partial^{2} R / \partial q^{2}\right]-R \cdot\left[\partial^{2} T / \partial p \partial q-\partial^{2} S / \partial q^{2}\right] \\
& -T \cdot\left[\partial^{2} S / \partial p^{2}-\partial^{2} R / \partial q \partial p\right] .
\end{aligned}
$$

We are thus led to distinguish two subcases $\mathrm{II}_{11}$ and $\mathrm{II}_{12}$ according as $D \not \equiv 0$ or $D \equiv 0$.

Case $\mathrm{II}_{11}$. In a neighborhood in which $\delta \neq 0$ and $D \neq 0,\left(U_{1} U_{2}\right) m$ $\equiv U_{6} m$ is not a linear combination of $U_{i} m(i=1,2,3,4,5)$, and upon adjoining $U_{6} m=0$ to our system we have a system of six independent equations in the partial derivatives of $m(x, y, z, p, q, M)$. Hence the most general integral of the system is a constant. Thus there exists no nonsingular solution of the system (5.2) and we have the following.

THEOREM 5.3. If $F_{3}=0$ does not have a self-adjoint equation of variation in a neighborhood in which $\delta \neq 0$ and $D \neq 0$, it admits no multiplier in this neighborhood.

The equation $p^{2} r+2 q^{2} s=0$ is an illustration of this case.

Case $\mathrm{II}_{12}$. In a neighborhood in which $\delta \neq 0$ and $D \equiv 0$ the system $U_{i} m=0(i=1,2,3,4,5)$ is complete and has $m=A\left(m_{1}\right)$ for its most general integral where $m_{1}(p, q, M)$ is a particular solution of $U_{i} m=0$ 
$(i=1,2)$ and $A$ is an arbitrary function of its argument. The most general nonsingular solution $M$ of the system (5.2) is obtained by solving for $M$ the relation $A\left(m_{1}\right)=$ const. The equation $M \cdot F_{3}=0$ now has a self-adjoint equation of variation and $M^{2} \cdot \delta \neq 0$. Hence, since multipliers which differ by a nonzero constant factor are not regarded as distinct, we have from Theorem 2.1 the following.

THEOREM 5.4. If $F_{3}=0$ does not have a self-adjoint equation of variation in a neighborhood in which $\delta \neq 0$ and $D \equiv 0$, then it admits as its most general multiplier in this neighborhood the unique solution for $M$ of the relation $A\left(m_{1}\right)=$ const.

The most general variation problem (I) can now be obtained in the usual manner. As an illustration of this case we cite the partial differential equation of minimal surfaces ${ }^{12}\left(1+q^{2}\right) r-2 p q s+\left(1+p^{2}\right) t=0$.

Case $\mathrm{II}_{2}$. If $\delta \equiv 0$ it follows that $R \cdot S \cdot T \not \equiv 0$, and hence in any neighborhood in which $R \cdot S \cdot T \neq 0$ we find that $U_{4} m \equiv(T / S) \cdot U_{3} m$. However, $U_{2} m \equiv(T / S) \cdot U_{1} m$ if and only if $\Delta \equiv \partial(R / S)^{2} / \partial q-2 \partial(R / S) / \partial p$ vanishes. We therefore consider the two subcases $\mathrm{II}_{21}$ and $\mathrm{II}_{22}$ according as $\Delta \equiv 0$ or $\Delta \not \equiv 0$.

Case $\mathrm{II}_{21}$. In a neighborhood in which $R \cdot S \cdot T \neq 0$ and $\Delta \equiv 0$, the system $U_{i} m=0(i=1,3)$ is equivalent to (5.4) and furthermore is complete. Hence we have the following result in this case.

THEOREM 5.5. If $F_{3}=0$ does not have a self-adjoint equation of variation in a neighborhood in which $R \cdot S \cdot T \neq 0, \delta \equiv 0$ and $\Delta \equiv 0$, then in this neighborhood $F_{3}=0$ admits as its most general multiplier the solution for $M$ of the relation $A\left(m_{1}, m_{2}, m_{3}, m_{4}\right)=$ const., where $m_{i}(i=1,2,3,4)$ are any four particular independent solutions of $U_{i} m=0(i=1,3)$ and where $A$ is different from zero but is otherwise an arbitrary function.

The equation $q^{2} r-2 p q s+p^{2} t=0$ is an illustration of this case.

Case $\mathrm{II}_{22}$. In a neighborhood in which $R \cdot S \cdot T \neq 0$ and $\Delta \neq 0$ we find it more perspicuous to consider the system (5.2). If in (5.2) we multiply the first equation by $S(p, q)$, the second by $R(p, q)$ and subtract, we obtain $M \cdot[S \cdot \partial S / \partial p-S \cdot \partial R / \partial q+R \cdot \partial S / \partial q-R \cdot \partial T / \partial p]=0$. Since $\Delta \neq 0$ we must have $M=0$, and hence the following is valid.

THEOREM 5.6. If $F_{3}=0$ does not have a self-adjoint equation of variation in a neighborhood in which $R \cdot S \cdot T \neq 0, \delta \equiv 0$ and $\Delta \neq 0$, then it admits no multiplier in this neighborhood.

The equation $p^{2} r+2 p q s+q^{2} t=0$ illustrates this case.

Fenn College

${ }^{12}$ Cf. L. LaPaz, Acta Szeged, vol. 5 (1932), pp. 199-207. 